\title{
EXTRAÇÃO DE COMPOSTOS BIOATIVOS DE FOLHAS DE MARACUJÁ-AMARELO (PASSIFLORA EDULIS SIMS)
}

\author{
C. I. SILVA ${ }^{1}$, J. C. C. SANTOS ${ }^{1}$, G. J. R. SOUZA ${ }^{1}$, L. F. OLIVEIRA ${ }^{1}$, T. P. V. B. DIAS ${ }^{1}$ \\ ${ }^{1}$ Universidade Federal de São João Del-Rei, Divinópolis/MG \\ E-mail para contato: miaismar@gmail.com
}

\begin{abstract}
RESUMO - A Passiflora edulis Sims é conhecida popularmente no Brasil como maracujá azedo ou amarelo, sendo muito utilizada na medicina popular e interessante para indústrias farmacêuticas e alimentícias, devido a sua propriedade calmante. $\mathrm{O}$ objetivo desse trabalho foi caracterizar os compostos bioativos nos extratos das folhas de Passiflora edulis Sims e comparar a eficiência de diferentes processos de extração. As amostras foram secas, moídas e, então, caracterizadas segundo sua densidade real e aparente, teor de voláteis e umidade, porosidade e diâmetro médio das partículas. Posteriormente, foram realizadas, extrações etanólicas (EE), etanólicas acedificadas (EAC) hidroalcoólicas (EH) e aquosas (EA) e em leito fixo, sendo quantificado nos extratos os compostos bioativos fenólicos, flavonoides e carotenoides. O pó das folhas de Passiflora edulis apresentou 2,30\% de umidade e voláteis, densidade aparente de $0,322 \mathrm{~g} / \mathrm{cm}^{3}$, densidade real de $1,1538 \mathrm{~g} / \mathrm{cm}^{3}$, porosidade média de 0,719 e diâmetro médio de partículas de $0,5487 \mathrm{~mm}$. As determinações dos rendimentos globais das extrações para EE, EAC, EH e EA, foram respectivamente de $5,0549 \%, 5,8791,13,2411 \%$ e $24,9192 \%$; os teores de flavonoides foram de 12,7842 mgQU/g, 7,8438 mgQU/g, 5,3865 mgQU/g e 2,1910 $\mathrm{mgQU} / \mathrm{g}$; de fenóis, 34,3948 $\mathrm{mgAG} / \mathrm{g}$, 35,3853 $\mathrm{mgAG} / \mathrm{g}, 24,8931 \mathrm{mgAG} / \mathrm{g}$ e $22,9080 \mathrm{mgAG} / \mathrm{g}$ e de carotenoides, 0,8278mg/100g, 0,7195mg/100g, 0,0823 e $0,2649 \mathrm{mg} / 100 \mathrm{~g}$. De modo geral, as extrações aquosas apresentaram maior rendimento global.
\end{abstract}

\section{INTRODUÇÃO}

A espécie Passiflora edulis Sims, popularmente conhecida como maracujá-azedo ou maracujá-amarelo, é encontrada por toda América do Sul, principalmente no Brasil (FIBGE, 2002). Pertencente à família Passifloraceae, é uma trepadeira perene de clima tropical, com folhas e flores largas e fruto comestível. O gênero Passiflora é o mais representativo da família, sendo composto por mais de 400 espécies, das quais 100 a 200 são brasileiras (Lopes, 1994).

O maracujá possui em sua constituição principalmente flavonoides, pectinas, passiflorina e benzoflavona. $\mathrm{O}$ composto bioativo com maior interesse para a indústria farmacêutica é o flavanóide, de estrutura polifenólica, com capacidade antioxidante e anti-inflamatória. Visando aumentar a eficiência para extração de compostos bioativos para sua utilização na indústria, o objetivo desse trabalho foi estudar o processo de extração dos compostos bioativos das folhas de Passiflora edulis Sims com a utilização de diferentes solventes, quantificando o valor de rendimento da extração e teor de fenóis, flavonoides e carotenoides nos extratos obtidos.

\section{METODOLOGIA EXPERIMENTAL}


As folhas de Passiflora Edulis Sims foram coletadas de uma plantação no município de Conceição das Pedras, Minas Gerais. A secagem das folhas foi realizada no LENGTEC (Laboratório de Engenharia de Processos e Tecnologias) em secador com circulação forçada de ar (Fabber, modelo 170, Piracicaba, Brasil) a $42{ }^{\circ} \mathrm{C}$ durante 72 horas. As amostras foram empacotadas em sacos plásticos, envoltas em papel alumínio e estocadas em freezer doméstico (modelo 220, Cônsul, Brasil) a $-10^{\circ} \mathrm{C}$.

A matéria-prima foi caracterizada quanto às propriedades das partículas (teor total de voláteis + umidade, diâmetro médio das partículas e densidade real) e propriedades do leito fixo de partículas (densidade aparente do leito de partículas e porosidade). Essas propriedades são importantes para a modelagem matemática e influenciam na curva de extração e no rendimento global das extrações.

\subsection{Caracterização da Matéria-prima}

Teor Total de Voláteis + Umidade (VU\%): Determinou-se o VU\% pela metodologia proposta por AOAC 930.04 (1997), onde $2 \mathrm{~g}$ de amostra foram pesados e levados à estufa à vácuo $(525 \mathrm{mmHg})$ a $105^{\circ} \mathrm{C}$ e secado até que a massa fosse $\leq 3 \mathrm{mg}$. Calculou-se o VU\% pela Equação 1:

$$
V U(\%)=\left[\frac{\text { massa inicial da amostra }- \text { massa final da amostra }}{\text { massa inicial da amostra }}\right] \times 100
$$

Diâmetro médio das partículas: utilizou-se o método proposto por ASAE (1997), onde a amostra foi disposta em um jogo de peneiras da série Tyler de tamanho de 8 a 48 mesh. Através da Equação 2, foi obtido o valor do diâmetro médio geométrico das partículas, que representa uma média ponderada dos diâmetros médios geométricos de cada fração (Equação 3).

$$
\begin{aligned}
& d_{m g}=\log ^{-1}\left[\frac{\sum_{i=1}^{n}(w i \log \bar{d} i)}{\sum_{i=1}^{n} w i}\right] \\
& \bar{d} i=\left(d_{i} \cdot d_{i+1}\right)^{1 / 2}
\end{aligned}
$$

Onde $\bar{d} i$ é o diâmetro médio geométrico, $d_{i}$ e $d_{i+1}$ representam a abertura da peneira $i$ e $i+1$, e $w_{i}$ é a massa do material retido na peneira $i$.

Densidade real do material triturado: A densidade real $\left(\rho_{r}\right)$ foi aferida utilizando picnômetros de $25 \mathrm{ml}$ e solvente tolueno (Mohsenin,1970), sendo calculada pela Equação 4:

$$
\rho_{r}=\frac{m_{(\text {picnômetro+amostra })-m_{(\text {picnômetro })}}}{v_{(\text {picnômetro })}-v_{(\text {tolueno })}}
$$

Densidade aparente do leito de partículas: A densidade aparente do leito $\left(\rho_{a}\right)$ foi obtida aferindo-se a massa de $P$. edulis trituradas em um volume de $10 \mathrm{~cm}^{3}$ (Uquiche et al, 2004).

Porosidade: A porosidade $(\varepsilon)$ do leito foi calculada pela Equação 5, utilizando-se dados obtidos de densidade aparente $\left(\rho_{a}\right)$ e real $\left(\rho_{r}\right)$ do material moído.

$$
\varepsilon=\frac{\text { volume do leito }- \text { volume ocupado pelo material }}{\text { volume do leito }}=1-\frac{\rho_{a}}{\rho_{r}}
$$




\subsection{Tipos de Extratos e Sistema Experimental}

Os extratos foram obtidos através de um extrator em leito fixo e de extração convencional. Os rendimentos das extrações foram calculados pela Equação 6:

$$
\text { Rendimento }=\frac{\text { Massa de extrato obtido final }}{\text { Massa de extrato bruto inicial }} \times 100
$$

Extração em leito fixo (supercrítico): Os experimentos foram conduzidos na unidade experimental do LENGTEC, UFSJ/CCO, Brasil. Ao extrator, com aproximadamente $100 \mathrm{~mL}$ de volume e diâmetro de $20 \mathrm{~mm}$, foi adicionado cerca de $10 \mathrm{~g}$ de folhas processadas, formando o leito de partículas. $\mathrm{O}$ volume restante foi completado com esferas de vidro de $5 \mathrm{~mm}$ cada. $\mathrm{O}$ banho termostatizado foi mantido entre $40{ }^{\circ} \mathrm{C}$ e $60^{\circ} \mathrm{C}$ e a pressão entre 100 bar e 200 bar. Aguardou-se um período fixo de 30 minutos para estabilização do sistema, e, então, iniciou-se a extração, escoando e coletando o extrato.

Extração convencional etanólica (EE): Os extratos foram obtidos segundo a metodologia descrita por Jorge (2008), com modificações. Com auxílio de um agitador magnético a extração ocorreu adicionando-se $10 \mathrm{~g}$ de folhas processadas e $50 \mathrm{~mL}$ de etanol em um erlenmeyer protegido da luz, por 90 minutos, em triplicata. O extrato foi filtrado e então o solvente foi evaporado em rotaevaporador acoplado a um banho a $40{ }^{\circ} \mathrm{C}$ sob vácuo de $700 \mathrm{mmHg}$ até a obtenção do extrato seco. As amostras foram armazenadas em frascos tipo penicilina de $50 \mathrm{~mL}$ lacradas e mantidas sob refrigeração, para análises posteriores.

Extração convencional etanólica acidificada (EAC): As EAC foram realizadas seguindo a metodologia para as EE, modificando-se, apenas, o solvente para uma solução formada por etanol $+0,3 \%$ de ácido cítrico.

Extração convencional hidroalcoólica (EH): Realizou-se as EH segundo a metodologia já descrita para as EE, modificando-se, apenas, o solvente para uma solução de etanol e água 70:30 (v:v).

Extração convencional aquosa (EA): Os extratos resultantes das EA foram obtidos com auxilio de um agitador magnético, adicionando-se, $10 \mathrm{~g}$ de folhas processadas e $100 \mathrm{~mL}$ de água em um erlenmeyer protegido da luz, por 90 minutos, em triplicata. $\mathrm{O}$ extrato foi filtrado e o solvente foi evaporado em estufa a vácuo $(490 \mathrm{mmHg})$ à temperatura de $40{ }^{\circ} \mathrm{C}$ até a obtenção do extrato seco. As amostras foram armazenadas em frascos tipo penicilina de $100 \mathrm{~mL}$ lacradas e mantidas sob refrigeração, para análises posteriores.

\subsection{Caracterização dos Extratos Obtidos (Métodos Analíticos)}

Compostos fenólicos: A determinação dos fenóis foi realizada segundo o método proposto por Singleton et al (1999).

Flavonoides totais: A determinação de flavonoides foi realizada segundo o método proposto pela Farmacopeia Brasileira (2010).

Determinação de carotenoides: A determinação de carotenoides foi realizada segundo o método proposto por Higby (1962). Sendo a quantidade de carotenoides determinada pela Equação 7:

$$
\text { Carotenoides }\left(\frac{m g}{100 g}\right)=\frac{A \times 10^{3}}{250 \times L \times W}
$$


Onde A corresponde a absorbância obtida no espectrofotômetro, L ao caminho óptico e W ao Coeficiente original entre amostra s inicial e o volume final da diluição.

\section{RESULTADOS E DISCUSSÃO}

\subsection{Caracterização da Matéria Prima}

As amostras de folhas processadas de Passiflora edulis Sims apresentaram um teor de voláteis + unidade (VU\%) de 2,30 $\pm 0,28$ e densidade real e aparente de $1,15 \pm 0,09$ e 0,322 $\pm 0,012$ $\mathrm{g} / \mathrm{cm}^{3}$, respectivamente, resultando uma porosidade de leito de $0,719 \pm 0,02$. O diâmetro médio de partículas foi $0,5487 \mathrm{~mm}$, formado pela média de material retido nas peneiras de meshes 8,12 , 16, 24, 32, 48 e fundo, com as porcentagens do material de diâmetros médios geométricos distribuídos como: 0,10\% (2,36 mm); 0,09\%(1,4 mm); 2,59\% (1 mm); 36,62\% (0,71 mm); $27,03 \%(0,5 \mathrm{~mm}) ; 19,45 \%(0,3 \mathrm{~mm})$ e $14,12 \%(0,15 \mathrm{~mm})$, respectivamente.

\subsection{Extrações}

Na Tabela 1 apresenta-se os dados obtidos para as amostras de Passiflora edulis Sims nas diferentes extrações realizadas.

Tabela 1 - Rendimento da extração, Teor de compostos fenólicos, Teor de flavonoides e Teor de carotenoides das amostras de Passiflora edulis Sims.

\begin{tabular}{ccccc}
\hline Extração & $\begin{array}{c}\text { Rendimento } \\
(\%)\end{array}$ & $\begin{array}{c}\text { Compostos } \\
\text { fenólicos } \\
(\mathrm{mgAG} / \mathrm{g})\end{array}$ & $\begin{array}{c}\text { Flavonoides } \\
(\mathrm{mgQU} / \mathrm{g})\end{array}$ & $\begin{array}{c}\text { Carotenoides } \\
(\mathrm{mg} / 100 \mathrm{~g})\end{array}$ \\
\hline Aquosa (EA) & $24,9192 \mathrm{c}$ & $22,9080 \mathrm{a}$ & $2,1910 \mathrm{a}$ & $0,2649 \mathrm{~b}$ \\
Hidroalcoólica (EH) & $13,2411 \mathrm{~b}$ & $24,8931 \mathrm{a}$ & $5,3865 \mathrm{a}, \mathrm{b}$ & $0,0823 \mathrm{a}$ \\
Etanólica (EE) & $5,0549 \mathrm{a}$ & $34,3948 \mathrm{a}$ & $12,7842 \mathrm{c}$ & $0,8278 \mathrm{c}$ \\
Etanol acidificado (EAC) & $5,8791 \mathrm{a}$ & $35,3853 \mathrm{a}$ & $7,8438 \mathrm{~b}$ & $0,7195 \mathrm{c}$ \\
\hline
\end{tabular}

Médias com letras iguais, em uma mesma coluna, não diferem entre si, pelo teste estatístico de Tukey

Os dados de rendimento da extração e de teor fenólico, flavonoides e carotenoides apresentados na Tabela 1 foram tratados estatisticamente com teste ANOVA, observando-se que os compostos fenólicos $(\mathrm{p}=0,1218)$ não diferiram significativamente para os quatro extratos analisados. Já os outros parâmetros analisados, apresentaram diferença ao nível de $5 \%$ de significância ( $\mathrm{p} \leq 0,0001$ ). Com base nos resultados da ANOVA, o teste de Tukey foi então realizado para as análises de rendimento das extrações, teor de flavonoides e teor de carotenoides.

Como podemos observar na Tabela 1, obteve-se um baixo rendimento global na EE $(5,0549 \%)$ e na EAC $(5,8791 \%)$, que não apresentaram diferenças significativas entre elas. No entanto, a mistura hidroalcoólica com $70 \%$ de etanol (EH) aumentou o rendimento para $13,2411 \%$, sendo o melhor rendimento, $24,9191 \%$, obtido para a EA. O baixo rendimento com etanol pode ser relacionado com sua volatilidade, onde o solvente evapora e perde-se ao longo do processo de extração e, consequentemente, há diminuição da concentração do mesmo na extração. Os melhores rendimentos foram encontrados nas extrações onde o solvente empregado era menos volátil (aquosa e hidroalcoólica), por manter sua concentração aproximadamente constante durante toda a extração. 
Segundo Diniz (2013), as extrações aquosas apresentam maior rendimento devido ao caráter polar do solvente. Em seus estudos, o autor sugere uma tendência crescente do rendimento da extração em favor do aumento da polaridade do solvente. Entretanto, não se pode inferir que um melhor rendimento também seja uma melhor extração de compostos bioativos.

Na Tabela 1, observa-se uma baixa quantidade de flavonoides nos extratos das EAC (7,8438 mgQU/g), EH (5,3865 mgQU/g) e EA (2,1910 mgQU/g), consideradas estatisticamente iguais, diferentemente do extrato da $\mathrm{EE}$, que apresentou uma concentração acima dos demais $(12,7842 \mathrm{mgQU} / \mathrm{g})$. Esta diferença pode ser explicada pelo fato de os flavonoides possuírem baixa afinidade com a água e segundo Simões et al. (2010), apresentarem maior solubilidade em solventes orgânicos.

A maior concentração de carotenoide foi encontrada nos extratos das EE e EAC $(0,8278$ e $0,7195 \mathrm{mg} / 100 \mathrm{~g}$ de amostra seca, respectivamente). Já nos extratos das EA e EH, a concentração foi menor $(0,2649$ e $0,0823 \mathrm{mg} / 100 \mathrm{~g}$ de amostra seca, respectivamente) devido a polaridade do solvente, já que carotenoides possuem baixa solubilidade em solventes polares como a água.

Dentre os compostos analisados, o de maior interesse neste trabalho são os compostos fenólicos. Por este motivo, nas extrações em leito fixo somente estes compostos foram analisados. Nessa extração, foi utilizado apenas água como solvente, pois como podemos observar na Tabela 1, as demais extrações não apresentaram diferenças significativas. Além disso a água é considerada um solvente não tóxico (tecnologia limpa). Os resultados obtidos são apresentados na Tabela 2.

Tabela 2 - Rendimento da extração e compostos fenólicos totais na extração de P. edulis em leito fixo e alta pressão.

\begin{tabular}{|c|c|c|c|}
\hline \multicolumn{2}{|c|}{ Ensaio } & \multirow{2}{*}{$\begin{array}{c}\text { Rendimento } \\
(\%)\end{array}$} & \multirow{2}{*}{$\begin{array}{c}\text { Teor de Fenóis } \\
\text { (mgAG/g de extrato) }\end{array}$} \\
\hline $\mathrm{T}\left({ }^{\circ} \mathrm{C}\right)$ & $\mathrm{P}\left(\mathrm{kgf} / \mathrm{cm}^{2}\right)$ & & \\
\hline 43 & 115 & 4,2260 & 362,7641 \\
\hline 57 & 115 & 17,4060 & 223,7320 \\
\hline 43 & 185 & 25,4230 & 213,3645 \\
\hline 57 & 185 & 6,0880 & 302,7896 \\
\hline
\end{tabular}

Na Tabela 2, observa-se que o rendimento obtido para a extração em leito fixo é aproximadamente igual ao rendimento obtido nas extrações convencionais, no entanto o teor de fenóis foi maior em todos os ensaios experimentais. Isso mostra que a temperatura e a pressão influenciam diretamente na quantidade de fenóis extraídos. A melhor condição de extração encontrada foi à temperatura de $43{ }^{\circ} \mathrm{C}$ e pressão de $115 \mathrm{kgf} / \mathrm{cm}^{2}$ com um teor de compostos fenólicos de 469,47 mgAG/g de extrato.

Segundo Simões et al. (2010), além de temperatura e pressão, a reposição de solvente extrativo proporciona uma extração mais eficiente, em comparação com a extração convencional, onde não há reposição de solvente. O extrator de leito fixo permitiu a obtenção de extratos de alta qualidade, com maior teor de fenóis e com rendimento igual ou pouco abaixo.

\section{CONSIDERAÇÕES FINAIS}

O estudo demostrou que as extrações para obtenção de compostos bioativos específicos em extrato de Passiflora Edulis Sims foram, em parte, mais eficientes com solventes pouco polares. 
Entretanto, para se determinar o solvente adequado, deve-se inicialmente definir qual a classe do composto de interesse a ser extraído.

$\mathrm{Na}$ análise de teor de compostos fenólicos totais, a mudança do solvente nos processos de extrações não apresentou diferença significativa, indicando que compostos fenólicos são um grupo com afinidade à solventes orgânicos e inorgânicos. Para o teor de flavonoides totais e carotenoides totais, o processo realizado com etanol e etanol acidificado obteve o melhor resultado.

O rendimento global da extração em leito fixo não mostrou diferenças em relação ao rendimento das extrações convencionais aquosas. Entretanto, o teor de fenóis encontrado foi superior as demais extrações, indicando maior eficiência no processo de extração desse composto. Isso se deve ao tempo de contato do solvente com as amostras e da não saturação das soluções.

\section{REFERÊNCIAS BIBLIOGRÁFICAS}

ANVISA; Farmacopeia Brasileira. v2. $5^{\mathrm{a}}$ ed. Brasilia.0 2010.

AOAC, Official Methods Of Analysis, 16th Edn. Washington, DC: Association Of Official Analytical Chemists. 1997.

ASAE, Standards. Method of Determining and Expressing Fineness of Feed Materials by Sieving. ASAE, S319.3, 547, 1997.

DINIZ, T. T. G; Obtenção de extratos de folhas de pitanga (Eugenia uniflora L.) e de alecrimpimenta (Lippia sidoides Cham.) por extração sequencial em leito fixo usando CO2 supercrítico, etanol e água. Dissertação - Universidade estadual de Campinas. Campinas, SP, 2013.

FONSECA, S. G. da C; Farmacotécnica de fitoterápicos. 2005.

FUNDAÇÃO DO INSTITUTO BRASILEIRO DE GEOGRAFIA E ESTATISTICA - FIBGE. Ministério da Integração Nacional. Secretaria da Infra-estrutura Hídrica. 80 Departamento de Desenvolvimento Hidroagrícola. Maracujá. Brasília, 2002, p. 1-2. (FrutiSéries, 2).

HIGBY, W. K; A Simplified method for determination of the carotenoid distribution in natural and carotene fortifield Orange Juice. Journal of food Science, 27, p.42-49. 1962.

JORGE, M. P; Atividade cicatrizante do estrato bruto de Arrabidaea chica (Humb. \& Bonpl). Verlot. Dissertação de mestrado; Faculdade de Ciências Médicas - Unicamp,Campinas, São Paulo. 2008.

LOPES, S. C. Citogenética do maracujazeiro - Passiflora spp. In: SÃO JOSÉ, A. R. (Ed.). Maracujá: produção e mercado. Bahia: Universidade Estadual da Bahia, 1994. p. 19-23.

MOHSENIN, N. R; Physical properties of plant and animal materials. $2^{\mathrm{a}}$ ed. New York: Gordon, 103 p., 1970.

SIMÕES, C. M. O; SCHENKEL, E. P; GOSMANN, G; Mello, J. C. P; MENTZ, L. A; PETROVICK, P. R; Farmacognosia: da planta ao Medicamento. 6ed ${ }^{\mathrm{a}}$, Porto Alegre: editora UFRGS. 2010.

SINGLETON, V. L; ORTHOFER, R; RAVENTOS, R. M. L; Analysis of total phenols and other oxidation substrates and antioxidants by means of folin-ciocalteu reagent. Methods in enzymology. V 199, p 152-178. 1999.

UQUICHE, E; DEL VALLE, J. M; ORTIZ, J; Supercritical carbon dioxide extraction of red pepper (Capsicum annuum L.) oleoresin, Journal of Food Engineering, v.65, n.1, p.55-66. 2004. 